\title{
Randomized Control Study for Evaluation of Effectiveness of Topical NSAIDS Patches to Prevent Backache Following Spinal Anesthesia in Caesarean Section
}

\author{
Aktham Adelshoukry*, Amrsobhy Abddelkway \\ Lecturer of Anesthesia and ICU, Anesthesia Department, Ain Shams University, Cairo, Egypt \\ Email: ^Aktham.shoukry1@gmail.com,dr.amrsobhy2013@gmail.com
}

How to cite this paper: Adelshoukry, A. and Abddelkway, A. (2018) Randomized Control Study for Evaluation of Effectiveness of Topical NSAIDS Patches to Prevent Backache Following Spinal Anesthesia in Caesarean Section. Open Journal of Anesthesiology, 8, 35-42.

https://doi.org/10.4236/ojanes.2018.81004

Received: December 11, 2017

Accepted: January 26, 2018

Published: January 29, 2018

Copyright $\odot 2018$ by authors and Scientific Research Publishing Inc. This work is licensed under the Creative Commons Attribution International License (CC BY 4.0).

http://creativecommons.org/licenses/by/4.0/

(c) (i) Open Access

\begin{abstract}
Background: Women who had caesarean section (CS) with spinal anaesthesia had more chances to develop low back pain. Controlled studies evaluating the prevention of back pain after neuraxial anaesthesia involved mostly the use of an anti-inflammatory medication or steroid, given epidurally or as part of the intradermal injectate. The purpose of this study is to determine the effect of topical Nonsteroidal Anti-inflammatory drugs (NSAIDS) patch on decreasing the incidence of post-operative backache following spinal anaesthesia for caesarean section. Patients and methods: One hundred thirty parturients aged 19 - 40 years, American society of Anaesthesiology class I \& II scheduled for elective caesarean section with spinal anaesthesia were divided randomly into two equal Groups (65 patients each): ketoprofen group (TDK) received $30 \mathrm{mg}$ ketoprofen transdermal patch at site of puncture for 24 hours and control group (TDC) received a placebo transdermal patch at site of puncture for 24 hours. In the Postoperative period 24 hours after the spinal procedure, the transdermal patch was removed in both groups then symptoms of back pain have been determined by visual numerical scale (VNS). Results: regard incidence of occurrence and severity of back pain $(\mathrm{VNS}>3)$ the results showed that the back pain was less in the TDK group than in the TDC group at 24 hours post spinal interval (4.6\% vs $15.4 \%$ ) respectively. On the other hand there was no statistical significant difference at 48 hours and 2 weeks post spinal interval. Conclusion: Prophylactic application of topical NSAIDS patch may reduce the incidence and the severity of short term backache after spinal anaesthesia in CS with negligible complications.
\end{abstract}

\section{Keywords}

Backache, Spinal Anaesthesia, Caesarean Section, NSAID Transdermal Patch 


\section{Introduction}

Backache following previous spinal anesthetic was the major cause for $13.4 \%$ patients refusing spinal anesthesia in a series of more than 1000 patients [1]. Although spinal anesthesia offers many advantages for cesarean section, it is not without complications, including transient neurological deficits. Women who had caesarean section with spinal anesthesia had more chances to develop low back pain than women with normal vaginal delivery [2].

Postoperative back pain could be of a short duration, lasting from 72 hours to a week or persistent, lasting beyond 3 months. Backache etiology associated with neuraxial blockade is thought to be multifactorial. It might be due to needle trauma, aseptic tendonitis, periosteitis, excessive stretching of ligaments after relaxation of Para spinal muscles with development of a supraspinous hematoma. Other contributing factors include type, duration of surgery, duration of immobilization, and the position of the patient during spinal puncture [3].

Epidural and intradermal anti-inflammatory medication or steroid, have been widely used in prevention of back pain after neuraxial anesthesia [4] [5] [6] [7]. The purpose of this study is to determine the effect of topical NSAIDS patch on decreasing the incidence of post-operative backache following spinal anesthesia for cesarean section.

\section{Patients and Methods}

This prospective randomized double blind placebo-controlled study was conducted on one hundred thirty parturients, aging between 19 and 40 years, ASA Class I \& II scheduled for elective cesarean section at the obstetric department of Ain Shams University Hospitals. The participants were enrolled from April 2017 to October 2017 after the approval of the Ethical Medical Committee and obtaining a written informed consent from them or their legal guardians.

Exclusion criteria included Parent's refusal, ASA III or more, previous history of sever back pain or surgery, Chronic recurrent back problems such as severe deformity of the spine, Contraindications to spinal anesthesia such as coagulopathy, allergy to local anesthetic, multiple spinal procedure attempt (more than two trials), loss of patient communication after discharge, duration of surgery longer than 2.5 hours, body mass index $\geq 32 \mathrm{~kg} / \mathrm{m}$.

For all enrolled parturients according to Ain shams Anesthesia preoperative assessment and routine laboratory investigations were done. Pre-induction all patients had an IV access (18 gauge peripheral cannula) using EMLA cream, premedication drugs were given in the form of intravenous $10 \mathrm{mg}$ metoclopramide and $50 \mathrm{mg}$ ranitidine, the circulation was preloaded with $500 \mathrm{ml}$ of $0.9 \%$ normal saline. Basic monitoring including ECG, pulse oximetry and non-invasive blood pressure was applied to all patients, through the whole procedure and in the PACU.

Spinal anesthesia was conducted under aseptic conditions using midline approach at the L2-3 or L3-4 interspace with the patient in the sitting position using 
a 25G Quincke spinal needle after local infiltration of the skin and subcutaneous

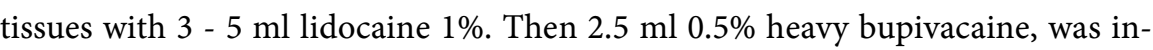
jected intrathecally by same blinded experienced anesthesiologist.

Patients were allocated randomly to one of two groups (65 patients each) using a closed sealed envelope method of randomization (Figure 1).

\section{Ketopfrofen Group (TDK Group)}

Receiveda transdermal $30 \mathrm{mg}$ ketoprofen (Kefentech) ${ }^{\circledast}$ patch at site of puncture for 24 hours.

\section{Control Group (TDC Group)}

Received aplacebo transdermal patch at site of puncture for 24 hours.

After confirming the adequate level of sensory blockade (between $T_{4}-T_{7}$ dermatomes) Cesarean section was initiated at the end of the procedure the patients were transferred to the PACU and then according to modified alderete score $\geq 9$ to the ward.

In the Postoperative period 24 hours after the spinal procedure, the intradermal patch was removed in both groups and all the patients were asked by blinded anesthetist; if they had experienced any of the symptoms of back pain in form of localized tenderness at the site of injection or pricking pain at the site of needle insertion or pain radiating to the buttocks.

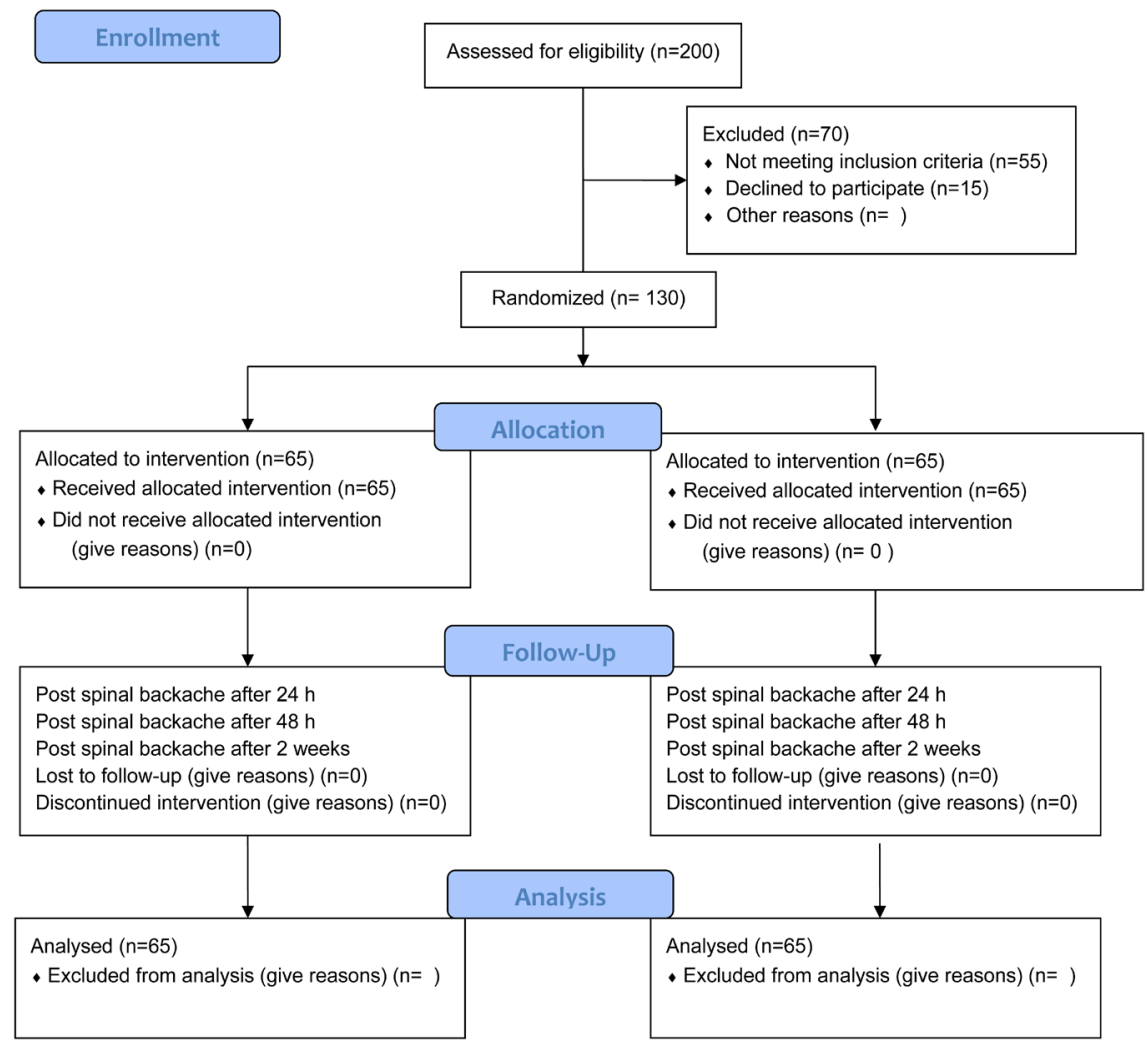

Figure 1. Flow diagram for randomized controlled trial (RCT) to our study. 
Assessment of pain was carried out using visual numerical scale (VNS) where end-point 0 denotes "no pain", 1 - 3 denotes "mild pain" [nagging, annoying, and interferes little with Activity of Daily Livings (ADLs)], 4 - 6 denotes "moderate pain" (Interferes significantly with ADLs) and 7 - 10 denotes "severe pain" (Disabling, unable to perform ADLs).

A patient was considered to have post spinal backache when VNS scale was $>3$.

Once hematoma and abscess have been ruled out, patients with moderate pain was managed by warm compresses or acetaminophen $1 \mathrm{gm} \mathrm{IV.}$

Postoperative pain management for both groups in first 48 hours was NSAID free regimen (I. M meperidine $50 \mathrm{mg}$ on demand) and the postoperative interviews were postponed at least 2 hours if patients had received meperidine within 1 hour.

Further assessment was done at another two time intervals: 48 hours postoperatively before hospital discharge and after two weeks in the postpartum visit. Finally the duration of surgery, the hospital length stay and complications as allergy or infection at the puncture site were also recorded.

\section{Statistical Methods}

With respect to sample size calculation. Based on VNS in the two groups. Group sample sizes of 65 each achieve $80 \%$ power to detect a difference of -0.8 (a drop in the VNS score) between the null hypothesis that both group means are 1.5 and the alternative hypothesis that the mean of controlled group is 2.3 with study group standard deviations of 1.5 with a significance level (alpha) of 0.05 using a two-sided Mann-Whitney test assuming that the actual distribution is uniform.

Statistical analysis was calculated using computer software statistical package for the social science (SPSS, version 20; SPSS Inc., Chicago, Illinois, USA) and was performed Description of quantitative (numerical) variables was performed in the form of mean \pm SD. Description of qualitative (categorical) data was performed in the form of number of cases and percent. Error bars represent $95 \%$ confidence interval. ANOVA test and chi-square tests was used for comparison among different times in the same group in quantitative data. The significance level was set at $P$-value of 0.05 or less, and $P$-value of 0.01 or less was considered highly significant.

\section{Results}

In this double blinded prospective study A total of 130 parturients were enrolled and randomly assigned to either study group with ketoprofen patch (TDK group) or control group with placebo patch (TDC group). No statistically significant difference between groups regarding demographics In terms of age, height, body weight of the patients, duration of surgery and hospital length of stay (Table 1).

The two groups as regard incidence of occurrence of back pain the results 
showed that the back pain was less on comparing the two groups as regard incidence of back pain the results showed that the back pain was less in the TDK group at 24 hour post spinal interval ( $4.6 \%$ vs $15.4 \%$ respectively). On the other hand there was no statistical significant difference at 48 hours and 2 weeks post spinal intervals (Table 2).

In addition the severity of back pain was compared between both groups. At 24 hours post spinal time interval the TDK group had less pain than the TDC group expressed in a low VNS score ( $3.77 \pm 1.14$ vs. $4.45 \pm 1.23$ respectively) with a statistically significant difference ( $P$-value 0.002$)$. However there was no significance in severity of pain when compared between both groups at 48 hours and, two weeks post spinal time intervals (Figure 2).

Analgesics consumption was not different between patients with or without post spinal backache. No other specific complications were noted during hospitalization. At follow up, no patient had developed local skin wound infection at the spinal needle puncture site.

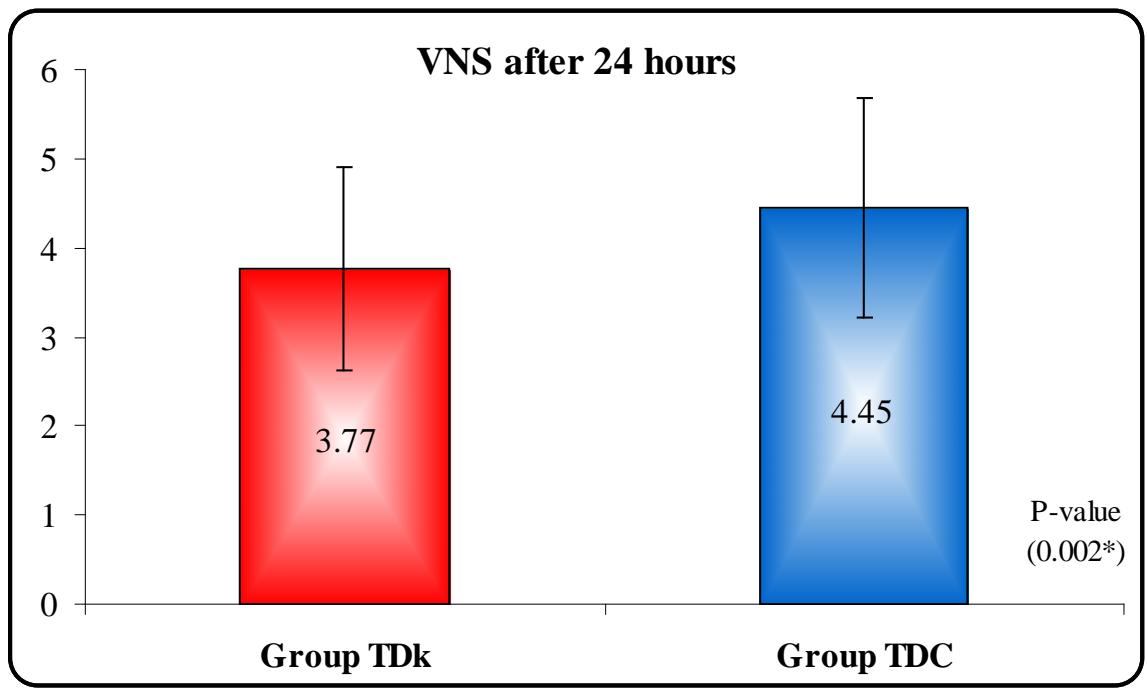

Figure 2. VAS after 24 hours post spinal. Group TDC: control group, Group TDK: ketoprofen patch group, VNS: visual numerical scale.

Table 1. Demographic data.

\begin{tabular}{ccccc}
\hline & $\begin{array}{c}\text { Group TDC } \\
(\mathbf{n}=65)\end{array}$ & $\begin{array}{c}\text { Group TDK } \\
(\mathbf{n}=65)\end{array}$ & \multicolumn{2}{c}{ T-test } \\
\cline { 2 - 5 } & Mean \pm SD & Mean \pm SD & $t$ & $P$-value \\
\hline Age (years) & $31.06 \pm 4.56$ & $29.84 \pm 4.15$ & 1.595 & 0.113 \\
Weight (kg) & $85.34 \pm 6.82$ & $84.25 \pm 7.34$ & 0.877 & 0.382 \\
Height (centimeter) & $167.75 \pm 5.37$ & $166.37 \pm 4.87$ & 1.535 & 0.127 \\
Duration of surgery (minute) & $85.63 \pm 4.81$ & $84.42 \pm 5.39$ & 1.350 & 0.179 \\
Postoperative length of stay (days) & $2.12 \pm 0.67$ & $2.05 \pm 0.51$ & 0.670 & 0.504 \\
\hline
\end{tabular}

Group TDC: control group, Group TDK: ketoprofen patch group, n: number, Kg: kilogram. 
Table 2. Incidence of occurrence of backache post spinal.

\begin{tabular}{ccccccc}
\hline & \multicolumn{2}{c}{$\begin{array}{c}\text { Group TDC } \\
\text { No of patient with backache }\end{array}$} & \multicolumn{2}{c}{$\begin{array}{c}\text { Group TDK } \\
(\mathbf{n}=65)\end{array}$} & \multicolumn{2}{c}{ Chi-square } \\
\cline { 2 - 7 } & $\mathrm{N}$ & $\%$ & $\mathrm{~N}$ & $\%$ & $X^{2}$ & $P$-value \\
\hline After 24 hours post spinal & 10 & 15.4 & 3 & 4.6 & 4.188 & $0.041^{*}$ \\
After 48 hours post spinal & 4 & 6.2 & 2 & 3.1 & 0.699 & 0.403 \\
After 2 weeks post spinal & 1 & 1.5 & 1 & 1.5 & 0.000 & 1.000 \\
\hline
\end{tabular}

Group TDC: control group, Group TDK: ketoprofen patch group, n: number, ${ }^{\star}$ statically significant.

\section{Discussion}

Backache is a common postoperative complaint after regional anesthesia. The present study demonstrated that prophylactic application of topical NSAIDS patch may reduce the incidence of post spinal backache after spinal anesthesia in cesarean section.

The etiology of backache associated with neuraxial blockade might be due to localized tissue trauma leading to aseptic periosteitis, tendonitis, inflammation of ligaments and osteochondritis. The tissue trauma initiates a cascade of events involving the arachidonic acid cycle. This includes the release of inflammatory mediators such as substance $\mathrm{P}$, histamine, bradykinin, prostaglandins, leukotrienes, and serotonin that sensitize their respective nociceptors producing peripheral hyperalgesia and inflammation [8].

Non-steroidal anti-inflammatory drugs inhibit prostaglandin synthase enzymes that convert the arachidonic acid to a series of prostaglandins, as a result both inflammation and hyperalgesia will decrease [9], therefore, the application of non steroidal at site of needle insertion may interrupt this cycle resulting in a decrease of occurrence of back pain hence increasing the overall patient comfort [7].

Patients were randomized into either a study group (TDK group) had received a ketoprofen intradermal patch at puncture site following spinal anesthesia or control group that received a intradermal placebo patch (free from drug) with 65 patients in each group. The patients were then evaluated for back pain 24, 48 hours and two weeks postoperatively using a $0-10 \mathrm{~cm}$ visual analogue scale. Based on the reported scores back pain was further classified as mild (1 - 3), moderate (4 - 6), or severe (7 - 10).

Our results showed significant decrease in incidence of backache occurrence in TDK group only 3 patients out of 65 expressed VAS score more than 3 (4.6\%) rather than 10 patients out of 65 (15.6\%) in TDC group while after 48 hours and two weeks following the spinal procedure there was no difference between both groups. This is consistent with a study by Hsieh et al. [6] found that local supplementation of ketoprofen reduced the incidence of low back pain after lumbar epidural analgesia. He used $4 \mathrm{~mL}$ of 1\% lidocaine with ketoprofen 1:400 for skininfiltration (study group) vs $4 \mathrm{~mL}$ of $1 \%$ lidocaine as control group before epidural needle placement. Then degree of back pain was then assessed using visual 
analogue scale at 24,48 , and $72 \mathrm{~h}$ following surgery. These investigators found that the control group reported a significantly higher ratio of back pain at all time interval measurements than did the study group.

Also, Todd and his colleague in 2002 [7] used ketorolac/lidocaine intradermal anesthesia ( $3 \mathrm{ml}$ of lidocaine $1 \%$ with $6 \mathrm{mg}$ ketorolac) during epidural catheter placement for labor analgesia. They found that intradermal ketorolac lidocaine combination given at the time of epidural catheter placement is beneficial in a reducing short term back pain in the postpartum period, especially in the event of cesarean delivery.

It should be noted that there were some limitations in our study, first the results didn't allow for an evaluation of the effectiveness of topical NSAIDS in all types of surgery, second we didn't study the overall cost effectiveness \& patients satisfaction regarding the use of these transdermal patches, finally we have only used the commercial available $30 \mathrm{mg}$ ketoprofen patch for 24 hours however since the postspinal backache inflammatory process is an ongoing process we recommend that further studies should be done using different doses of transdermal NSAIDS for longer periods more than 24 hours.

We conclude that prophylactic application of topical NSAIDS patch may reduce the incidence and the severity of short term backache after spinal anesthesia in cesarean section especially the in first 24 hours with negligible complications.

\section{References}

[1] Rafique, M.K. and Taqi, A. (2011) The Causes, Prevention and Management of Post Spinal Backache: An Overview (Review Article). Anaesthesia, Pain \& Intensive Care, 15, 65-69.

[2] Joshi, A.K. and Joshi, C. (2016) Comparative Study of Occurrence of Postpartum Low Back and Pelvic Pain (LBPP) after Normal Delivery versus Caesarean Section (CS) Following Spinal Anaesthesia and Its Rehabilitative Management. International Journal of Therapies and Rehabilitation Research IJTRR, 5, 4. https://doi.org/10.5455/ijtrr.000000139

[3] Schwabe, K. and Hopf, H.B. (2001) Persistent Back Pain after Spinal Anaesthesia in the Non-Obstetric Setting: Incidence and Predisposing Factors. British Journal of Anaesthesia, 86, 535-539. https://doi.org/10.1093/bja/86.4.535

[4] Wang, Y.L., Tan, P.P., Yang, C.H., Tsai, S.C. and Chung, H.S. (1997) Epidural Dexamethasone Reduces the Incidence of Backache after Lumbar Epidural Anesthesia. Anesthesia \& Analgesia, 84, 376-378. https://doi.org/10.1213/00000539-199702000-00025

[5] Wang, Y.L., Hsieh, J.R., Chung, H.S., Yu, C.L., Ho, A.C., Lu, P.P. and Tan, P.P. (1998) The Local Addition of Tenoxicam Reduces the Incidence of Low Back Pain after Lumbar Epidural Anesthesia. Anesthesiology, 89, 1414-1417. https://doi.org/10.1097/00000542-199812000-00019

[6] Hsieh, J.R., Hui, Y.L., Yu, C.C., Lau, W.M., Ng, Y.T. and Wang, Y.L. (1999) Local Supplementation of Ketoprofen Reduces the Incidence of Low Back Pain after Lumbar Epidural Anesthesia. Chang Gung Medical Journal, 22, 439-444

[7] Todd, G., John, A., Vacchiano, C. and Pellegrini, J. (2002) Intradermal Ketorolac for 
Reduction of Epidural Back Pain. International Journal of Obstetric Anesthesia, 11, 100-104. https://doi.org/10.1054/ijoa.2001.0936

[8] Ben-David, B., Katz, E., Gaitini, L. and Goldik, Z. (1995) Comparison of i.m. and Local Infiltration of Ketorolac with and without Local Anesthetic. British Journal of Anaesthesia, 75, 409-412. https://doi.org/10.1093/bja/75.4.409

[9] Reuben, S., Steinberg, R., Kreitzer, J. and Duprat, K. (1995) Intravenous Regional Anesthesia Using Lidocaine and Ketorolac. Anesthesia \& Analgesia, 81, 110-113. 